\title{
A Study of the Persistence of Mycobacterium bovis in the Environment under Natural Weather Conditions in Michigan, USA
}

\author{
Amanda E. Fine, ${ }^{1,2}$ Carole A. Bolin, ${ }^{3}$ Joseph C. Gardiner, ${ }^{4}$ and John B. Kaneene ${ }^{1}$ \\ ${ }^{1}$ Department of Large Animal Clinical Sciences and Center for Comparative Epidemiology, College of Veterinary Medicine, \\ Michigan State University, East Lansing, MI 48824-1314, USA \\ ${ }^{2}$ Wildlife Conservation Society, P.O. Box 485, Post Office 38, Ulaanbaatar 211238, Mongolia \\ ${ }^{3}$ Diagnostic Center for Population and Animal Health, Michigan State University, 4125 Beaumont Road, Lansing, \\ MI 48910-8104, USA \\ ${ }^{4}$ Department of Epidemiology, College of Human Medicine, Michigan State University, B601 West Fee Hall, East Lansing, \\ MI 48824, USA
}

Correspondence should be addressed to John B. Kaneene, kaneene@cvm.msu.edu

Received 4 January 2011; Accepted 16 February 2011

Academic Editor: Mitchell V. Palmer

Copyright (C) 2011 Amanda E. Fine et al. This is an open access article distributed under the Creative Commons Attribution License, which permits unrestricted use, distribution, and reproduction in any medium, provided the original work is properly cited.

Reisolation of Mycobacterium bovis from inoculated substrates was used to follow the persistence of viable M. bovis bacteria exposed to natural weather conditions over a 12-month period. Environmental factors were recorded continuously, and factors affecting M. bovis persistence (i.e., temperature, season, and substrate) were studied using survival analysis and Cox's proportional hazards regression. Persistence of M. bovis in the environment was significantly shorter in the spring/summer season, characterized by the highest average daily temperatures over the 12-month period. M. bovis persisted up to 88 days in soil, 58 days in water and hay, and 43 days on corn. These studies demonstrate that $M$. bovis bacteria persist long enough to represent a risk of exposure for cattle and/or wildlife and strengthen evidence that suggests cattle farm biosecurity and efforts to eliminate supplemental feeding of white-tailed deer will decrease the risk of bovine TB transmission among and between cattle and deer populations.

\section{Introduction}

An endemic focus of bovine tuberculosis (TB), caused by a single strain of Mycobacterium bovis, has been identified in white-tailed deer (Odocoileus virginianus) in northeast lower Michigan [1-3]. Spillover of M. bovis infection from whitetailed deer to cattle is suspected in the majority of the 52 cattle farms in the same region of the State identified as bovine TB positive since intensive surveillance for TB in Michigan livestock was reinitiated in 1998 (Michigan Department of Agriculture; USDA/APHIS/VS). The emergence of a wildlife reservoir for bovine TB in Michigan, and evidence of disease transmission between infected free-ranging white-tailed deer populations and domestic cattle, has forced a reevaluation of the understanding of the epidemiology of bovine $\mathrm{TB}$ in North America. Disease transmission between deer and cattle in Michigan is thought to occur in the absence of close contact between the species [4]. This has raised questions about the role of indirect transmission of M. bovis in the epidemiology of bovine TB and identified a need to investigate the persistence of $M$. bovis in the environment and the potential role of contaminated substrates in the transmission of M. bovis among and between wildlife and cattle populations.

The persistence of $M$. bovis in the environment and role of indirect transmission in the epidemiology of bovine TB in Michigan has been debated since the current TB epidemic in Michigan was first described in 1997 [2]. Bovine TB infection in white-tailed deer today is likely linked to the large number of cattle infected with $M$. bovis in Michigan during the late 1950's [5], however, the establishment and persistence of $M$. bovis in free-ranging white-tailed deer in northeast Michigan is thought to have been influenced by the longterm practice of Winter feeding of deer in the region [2]. 
These piles of feed, set out to attract deer and improve their productivity and Winter survival, are thought to contribute to the transmission of $\mathrm{TB}$ among white-tailed deer by (1) increasing local density and contact between animals and (2) providing a site for the indirect transmission of TB through contamination of the feed by infected deer shedding $M$. bovis in their saliva or nasal discharges and the subsequent infection of a naïve deer by consumption of contaminated feed $[2,6]$. This is supported by evidence that suggests that specific supplemental feeding practices, generally indicative of large-scale feeding operations, are associated with an increasing risk for bovine TB in deer in Michigan [7].

The role of $M$. bovis contaminated environmental substrates in the interspecies transmission of bovine TB between cattle and deer has also been investigated [8, 9]. Although $M$. bovis was not identified from any of the environmental substrates tested, particular cattle management practices and environmental factors were identified that have been shown to be associated with tuberculosis on cattle farms in northeast Michigan in the past [10]. These practices likely facilitate the indirect transmission of bovine TB from deer to cattle via $M$. bovis contaminated substrates. The factors and practices identified included the presence of ponds or open water in cattle areas, maintaining cattle outside more than $50 \%$ of the time, feeding, and watering cattle outside and not protecting feed intended for cattle from deer.

Evidence suggests that opportunities for the indirect transmission of $M$. bovis between white-tailed deer and cattle exist in northeast Michigan under current cattle and deer management practices. Data on the persistence of $M$. bovis on various environmental substrates and the factors that influence its survival are essential to the further understanding of the complexity of bovine TB transmission and epidemiology in Michigan. In addition to contributing to our understanding of bovine TB dynamics in this system, information regarding the persistence of $M$. bovis in the environment will support efforts to improve protocols for cattle farm biosecurity and the maintenance of appropriate restrictions on feeding and baiting free-ranging white-tailed deer and other wildlife.

Experimental studies conducted in New Zealand, Australia, South Africa, Great Britain, and Ireland, have shown that $M$. bovis persists in typical environmental substrates for varying amounts of time [11-15].

This study was designed to describe the persistence of the Michigan strain of M. bovis in typical environmental substrates (corn, hay, soil, and water) exposed to natural weather conditions in Michigan. Factors affecting the length of persistence, or survival time, of $M$. bovis in the environment were also investigated. The objective of the study was to determine whether or not $M$. bovis can survive in environmental substrates for sufficient lengths of time to serve as a source of infection for cattle and/or wild deer.

\section{Materials and Methods}

2.1. Culture, Media, and Growth Conditions. A Michigan strain of Mycobacterium bovis was obtained from a frozen culture of $M$. bovis originally isolated in 2002 from the retropharyngeal lymph node of a naturally infected 2year-old Holstein cow from Michigan. The animal was classified as a reactor on a comparative cervical test and had gross and microscopic lesions consistent with bovine TB at necropsy. The frozen $M$. bovis culture was added to $10 \mathrm{~mL}$ of Middlebrook $7 \mathrm{H} 9$ Broth with Middlebrook ADC Enrichment for cultivation of mycobacteria (BectonDickinson, Cockeysville, Md, USA). Multiple $10 \mathrm{~mL}$ vials of $M$. bovis inoculated $7 \mathrm{H} 9$ Broth were incubated at $37^{\circ} \mathrm{C}$ for 21 to 30 days. The exact final concentration of colony forming units of $M$. bovis liquid culture stock $(\mathrm{CFU} / \mathrm{mL})$ was determined by monitoring turbidity and then performing colony counts on Selective 7H11 agar (Becton-Dickinson) plates inoculated with exactly $100 \mu \mathrm{L}$ of $M$. bovis liquid stock, a $1: 10,1: 100,1: 1000$ and a $1: 10000$ dilution. Blood and CNA agar plates were inoculated with $M$. bovis liquid culture stock to monitor for contamination.

2.2. Environmental Substrates. Substrates selected for testing included grass hay, soil, water, and shelled corn. Grass hay was collected from the feed storage area of the Michigan State University (MSU) Large Animal Veterinary Teaching Hospital in East Lansing, Mich, USA. Soil was collected from the Baker Woodlot (Rachana Rajendra Neotropical Bird Sanctuary) located in the south central section of the MSU campus. Water was collected from the large pond at the center of the Baker Woodlot and from the Red Cedar River at the Farm Lane Bridge on the MSU campus. Shelled corn was purchased in 20 -pound $(\sim 9 \mathrm{~kg})$ bags from a local feed store. Environmental substrates were stored at $4^{\circ} \mathrm{C}$ with no exposure to light.

A set of environmental substrates consisted of 4 samples each of grass hay, soil, water, and shelled corn for a total of 16. Ball Half-Pint (236 mL) Regular Can-or-Freeze Jars were filled with $5 \mathrm{gm}$ of hay, $10 \mathrm{gm}$ of soil, $10 \mathrm{gm}$ of corn, or $10 \mathrm{~mL}$ of water. Half of the sample-filled jars were autoclaved for 2 hours at $121^{\circ} \mathrm{C}$ and 20 psi to sterilize the contents. Each sample set of 16 was identified with uniquely colored tape and a label denoting the sample type, sample set number, the autoclave status, and shade or nonshade treatment.

2.3. Facility (Laboratory and Outdoor Enclosure). Mycobacterium bovis sample inoculation, sample processing, and $M$. bovis isolation procedures were all performed in the biosafety level III (BL3) laboratory in the Diagnostic Center for Population and Animal Health (DCPAH) at Michigan State University (MSU).

The M. bovis environmental persistence studies were carried out in a structure erected on a concrete slab along the north fence of the livestock containment facility south of the DCPAH at MSU. The structure consisted of an enclosed "cage" $4.88 \mathrm{~m} \times 7.62 \mathrm{~m} \times 2.44 \mathrm{~m}$ with a galvanized steel frame covered with $2.44 \mathrm{~mm}$ fencing (chain link-type fence with $2.44 \mathrm{~mm}$ holes) on all sides including the top. The bottom rail of the cage was flush with the concrete slab or buried below the ground surface. Any gaps below the bottom rail were closed with $0.61 \mathrm{~m} \times 1.22 \mathrm{~m}$ wooden beams. A 


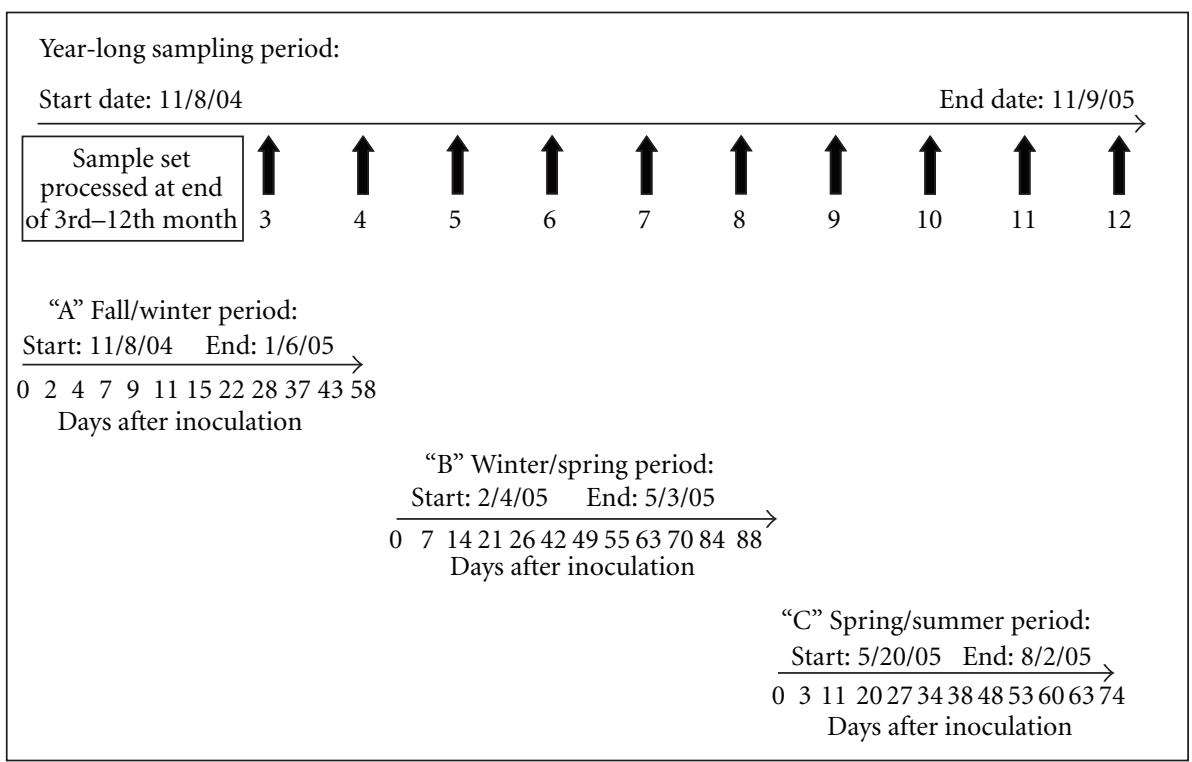

FIGURE 1: Timing of processing samples after M. bovis inoculation over year-long and seasonal sampling periods.

locked door was built into one side of the enclosure with a minimum clearance with the concrete slab and doorframes.

The fencing excluded all birds and small mammals. The structure was built to exclude livestock and/or deer in the unlikely event that they gain entrance to the fenced containment facility and access to the experimental enclosure was limited to authorized individuals.

Specified sets of $M$. bovis inoculated environmental substrates were placed within secondary clear plastic containers on 2 lines of steel tables set up within the enclosure. Sample containers on 1 line of steel tables were covered with black shade cloth. All secondary sample containers were lined with gravel and sand and secured with wire mesh covers.

2.4. Environmental Monitoring. A WeatherHawk weather station, Division of Campbell Scientific, Inc., was positioned at the center of the enclosure. The station was powered by a solar panel charged battery pack. Environmental data collected included rainfall $(\mathrm{mm})$, wind speed $(\mathrm{m} / \mathrm{sec})$, temperature $\left({ }^{\circ} \mathrm{C}\right)$, humidity level $(\%)$, and solar radiation $\left(\mathrm{W} / \mathrm{m}^{2}\right)$. Evapotranspiration, a combination of solar radiation, temperature, wind speed, and humidity, was also calculated with the WeatherHawk Virtual Weather software. The weather station was programmed to record data at 20-minute intervals, 24 hours a day. Environmental data was downloaded from the weather station to an Excel (Microsoft Corporation, Redmond, Wash, USA) spreadsheet on a desktop computer using a wireless system. Temperature and precipitation data were also collected over the same time periods from a weather station in the Michigan Automated Weather Network (MAWN) in Hawks City, Presque Isle County, Mich, USA in the bovine TB area.

2.5. Inoculation with $M$. bovis. Each substrate sample was inoculated with 50,000 CFUs of a strain of Mycobacterium bovis originally isolated from the lymph node of a cow in Michigan that tested positive for bovine TB and presented at necropsy with pathologic lesions typical of $M$. bovis infection. Samples were inoculated in the BL3 laboratory. Sample jars were then sealed with plastic, leak-proof lids and transported to the outdoor experimental enclosure, located 500 meters from the BL3 laboratory, in sealed and labeled coolers. Each sample set was then placed in the secondary containers within the enclosure described above. The plastic lids were removed from each sample jar and the wire mesh was placed over the secondary containers and secured.

2.6. Study Design and Sampling. The persistence of M. bovis in environmental substrates was evaluated over 4 sampling periods as presented in Figure 1. For each sampling period, 12 sets of 16 samples ( 4 grass hay, 4 corn, 4 water, and 4 soil) were inoculated with $M$. bovis which allowed for sample processing for $M$. bovis isolation at time 0 and at 11 additional time points. The first sample period spanned 12 months. Samples were placed in the enclosure on November 8 , 2004. Sample sets were processed monthly with the final set processed on November 9, 2005. Three additional sampling periods were nested within the sampling year to allow for the collection of seasonal data. The first seasonal sampling period " $\mathrm{A}$ " was for 58 days during the Fall/Winter (November 8, 2004-January 6, 2005). The second sampling periods " $\mathrm{B}$ " was for 88 days during the Winter/Spring (February 4, 2005-May 3, 2005). The third sampling periods "C" was for 74 days during the Spring/Summer (May 20, 2005-August 2, 2005), and a set of samples was processed monthly from November, 2004 to December, 2005. At the start of each seasonal sampling period twelve sets of $16 \mathrm{M}$. bovis inoculated samples were placed in the environmental sample enclosure (Figure 2). The persistence of $M$. bovis over time was determined by processing the sample sets for 


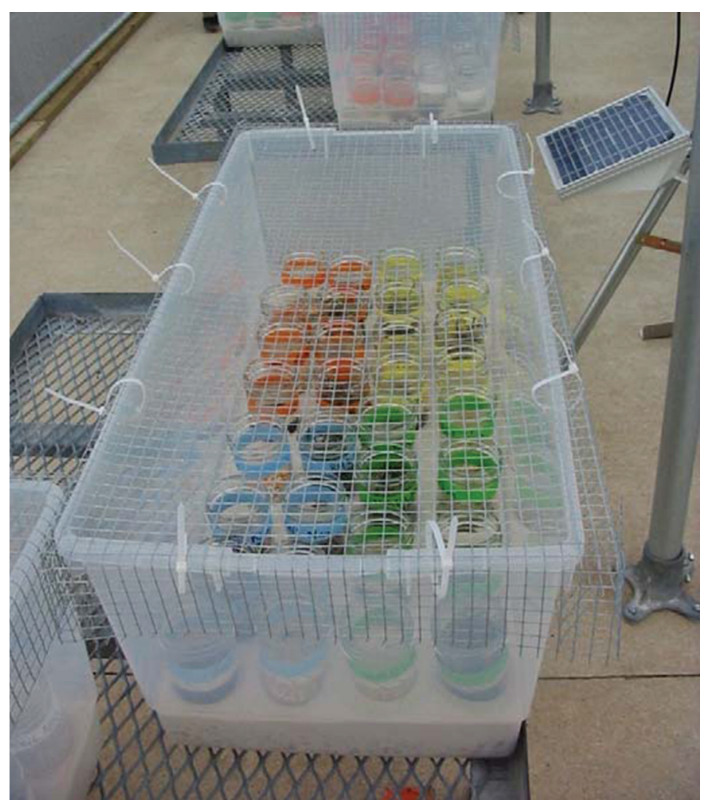

Figure 2: Photograph of samples of $M$. bovis inoculated substrates in their primary containers placed within their secondary containers inside the experimental environmental sample enclosure. Shown here are the "nonshaded" treatments from 4 sample sets (total of 32 sample jars).

mycobacterial culture and attempting to isolate $M$. bovis at time 0 and 11 additional time points. The size of the environmental sample enclosure limited the number of sample sets that could be placed in the enclosure at any given time which limited the number of sets per sampling period to 12 . The schedule for sample set processing was varied slightly from season to season in an attempt to capture both the pattern of $M$. bovis die off over time (number of colony forming units isolated from $100 \mu \mathrm{L}$ of processed sample) and the time point of the last positive and first negative M. bovis culture from a processed sample recorded for a particular sample substrate. As indicated in Figure 1, sample sets from the "Fall/Winter" period were collected every other day for 2 weeks and then weekly over 58 days, sample sets from the "Winter/Spring" were collected weekly over 88 days and sample sets from the "Spring/Summer" were collected on the third day and then approximately weekly over a 74 day period.

2.7. Environmental Sample Processing. At the scheduled sampling point a set of samples was collected from the environmental sample enclosure and transported (with lids replaced and in a sealed cooler) to the BL3 laboratory for processing. Sample substrates were allowed to come to room temperature before processing if frozen at collection. All samples were processed using the TB Culture Kit with Lytic Decon II (Integrated Research Technology, LLC, Quest Diagnostics Inc., Baltimore, $\mathrm{Md}$ ) and a protocol standardized by Fine et al. for processing environmental samples (hay, soil and water) for mycobacterial culture [16]. Collected samples were processed within their original container (Ball 1/2 pint $(236 \mathrm{~mL})$ regular jar). If necessary, as was the case with

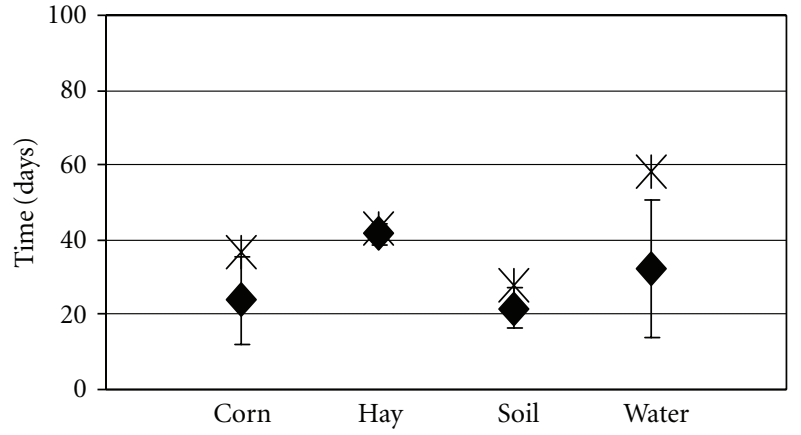

(a)

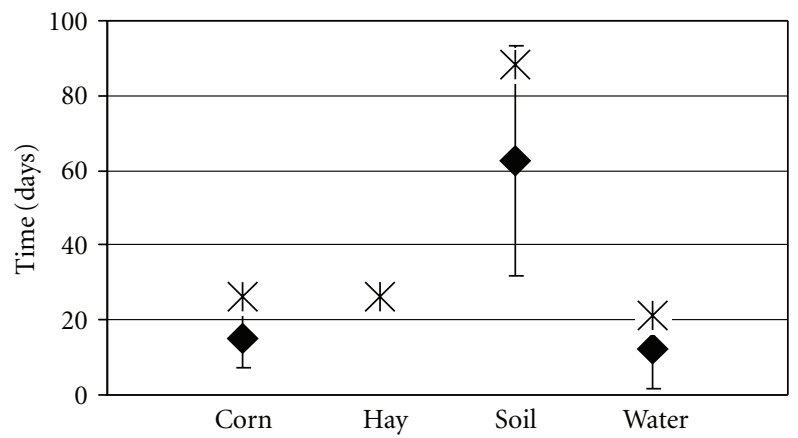

(b)

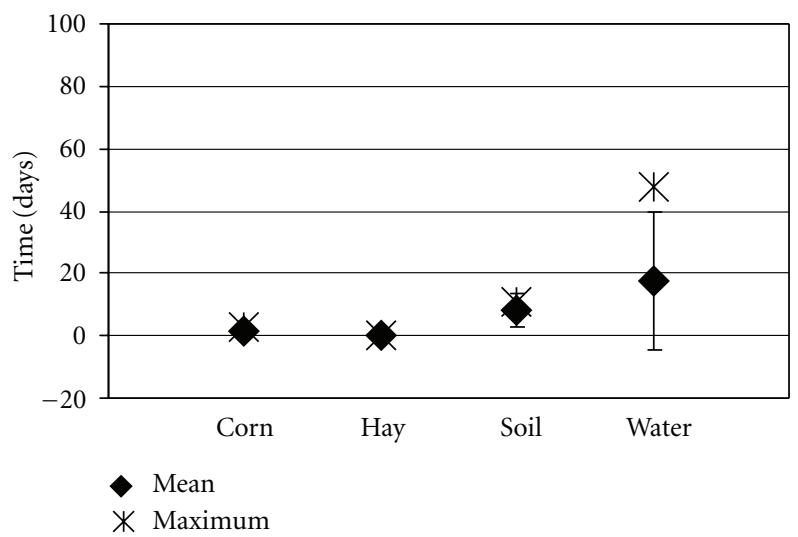

(c)

Figure 3: The mean and maximum survival of $M$. bovis in corn, hay, soil, and water exposed to environmental conditions in Fall/Winter (a), Winter/Spring (b), and Spring/Summer (c). Error bars represent standard deviation.

desiccated samples, sterile water $(5-10 \mathrm{~mL})$ was added to the solid substrates. Samples were pulverized and homogenized by securing a blade unit and gasket on the jars, inverting and blending them for 30 seconds on high with a household blender.

The samples were placed upright and allowed to settle for 30 minutes. The top $5 \mathrm{~mL}$ of fluid from each sample was removed and transferred to a $50 \mathrm{~mL}$ conical tube containing $10 \mathrm{~mL}$ of Decontamination Solution (20X Tris-citrate Buffer, CB-18 Stock, NALC, and water). Samples were mixed with a vortex machine and incubated at $37^{\circ} \mathrm{C}$ for 75 minutes. Sterile water was added to the $50 \mathrm{~mL}$ mark on each tube, 
mixed and centrifuged at 3,000 g for 20 minutes. Pelletcontaining tubes were decanted completely. A pipette was used to remove all but $1-3 \mathrm{~mL}$ of liquid from samples without a visible pellet. The pellet was resuspended in the supernatant backwash. One $\mathrm{mL}$ of sterile water was added and mixed. A $0.5 \mathrm{~mL}$ sample was transferred to a $2.0 \mathrm{~mL}$ labeled cryogenic vial and frozen at $-80^{\circ} \mathrm{C}$ and maintained for additional experiments or follow-up testing. One mL of 2X Resuspension Solution (10X-Enzyme StockTrichoderma harzianum extract, lysozyme and Lysobacter extract, and NALC) was added to each sample and samples were incubated for 45 minutes at $37^{\circ} \mathrm{C}$.

2.8. Mycobacterial Culture and Isolation. CB-18 processed samples were inoculated onto solid 7H11 Selective plates (Becton-Dickinson) by transferring $100 \mu \mathrm{L}$ of processed sample onto the plates and spreading the sample equally across the media with individual L-shaped spreaders. Two culture replicates were set up for each sample and the plates were incubated at $37^{\circ} \mathrm{C}$ for 12 weeks and examined weekly for colony formation. Typical M. bovis colonies were counted and recorded on laboratory data sheets. Acid-fast smear analysis was performed to confirm the presence of acidfast bacteria using standard protocols for slide preparation, staining, and examination [17]. A subset of the acidfast positive isolates was confirmed to be Mycobacterium tuberculosis complex species group using a genetic probe (AccuProbes, Gen-Probe, San Diego, Calif, USA).

2.9. Data Analysis. Mycobacterial culture results, recorded on laboratory data sheets for each sampling period, were entered in an Excel spreadsheet (Excel, Microsoft Office XP Professional, Redmond, Wash, USA). Data from the weather records and mycobacterial culture results were imported into SAS software (SAS version 9.0, SAS Institute, Inc., 2003, Cary, NC, USA) and combined. Summaries of the weather records for each sampling period were created and descriptive statistics were generated for the persistence of $M$. bovis on each sample type for all sampling periods. Statistical analyses were carried out with SAS software.

Differences between mean $M$. bovis survival time (persistence) in shade/nonshade treated samples and sterilized/nonsterilized treated substrates were compared using $t$ tests. To test for significant differences in the mean survival time (persistence) in sample types and sampling seasons (Fall/Winter, Winter/Spring, and Spring/Summer), we used analysis of variance (ANOVA) with the Bonferroni adjustment of $P$-values for multiple comparisons. Assessment of associations between the bovine TB status of experimental samples (positive versus negative) and covariates (sample type, shade/nonshade, and sterilized/nonsterilized substrate) were conducted using the Cochran-Mantel-Haenszel Chisquare tests or 2-tailed Fisher's exact test where appropriate.

Survival analyses were used to compare the persistence of $M$. bovis in the environment between seasons and to study the effects of environmental factors on $M$. bovis survival across the seasons. The time from sample inoculation to the first negative bovine $\mathrm{TB}$ culture after the last positive bovine TB culture was used as the survival time, or $M$. bovis persistence period, for each sample. The Log rank and Wilcoxon tests were used to compare the survival distributions for M. bovis persistence in the environment across the 3 seasons tested. The survival function or Kaplan-Meier curves for $M$. bovis persistence in each of the three seasons and in each of the 4 substrates across the three seasons were plotted. Cox's proportional hazards regression was used to study the effects of the nonweather-related covariates (sample type, shade/nonshade, and sterilized/nonsterilized substrate) and season on $M$. bovis survival in the environment.

Model selection in Cox regression was used to identify specific weather or seasonal factors that influenced the survival of $M$. bovis in the environment. The weather data were summarized as daily means, maxima and minima for rainfall, wind speed, temperature, humidity, barometer readings, solar radiation, and evapotranspiration. Spearman correlation coefficients $(r)$ were computed to identify potential areas of multicollinearity between the weather-related risk factors.

A multivariable Cox proportional hazards regression model was developed based on an initial evaluation of univariable models for each weather-related risk factor. The model contained all weathered-related risk factors that were significant at $P$-value $<.15$ on the likelihood ratio test (LRT). Highly correlated weather-related risk factors were removed due to redundancy of information and multicollinierity (e.g., solar radiation and evapotranspiration were both highly correlated with temperature). Purposeful selection of covariates and a modified stepwise method of variable evaluation with the entry $P$-value of .15 and the "stay" or removal $P$-value of .20 were used to build the final multivariable model for M. bovis survival in the environment.

\section{Results}

Detailed results from $M$. bovis inoculated substrates are presented in this section for the three sampling periods outlined in Figure 1 representing the seasons " $A$ " Fall/Winter, "B" Winter/Spring and "C" Spring/Summer. Detailed results are not provided for the year-long sampling period outlined in Figure 1 because no M. bovis bacteria was isolated from samples processed at months three through twelve. The initial 2 months of the 12-month sampling period was covered by sampling period " $\mathrm{A}$ " Fall/Winter, with data on weather conditions presented in Table 1 and data on M. bovis persistence presented in Table 2 .

3.1. Mycobacterial Culture. One hundred and ninety-two sample replicates from each of the three sampling periods (total 576) were processed for M. bovis isolation. Contamination of cultures with mold and other nonmycobacterial species was detected in $13 \%$ of the samples processed in sampling period Fall/Winter " $\mathrm{A}$ " and Winter/Spring " $\mathrm{B}$ " and $50 \%$ of the samples processed in sampling period Spring/Summer "C". Sample substrates that were sterilized before $M$. bovis inoculation had significantly lower odds of 
TABLE 1: Weather conditions recorded over the three sampling periods at the site of the Mycobacterium bovis environmental persistence study and temperature and precipitation data from the TB area of Michigan for the same time.

\begin{tabular}{lccc}
\hline & Fall/Winter “A” 11/8/04-1/6/05 & Winter/Spring “B” 2/4/05-5/3/05 & $\begin{array}{c}\text { Spring/Summer “C” 5/20/05-8/2/05 } \\
\text { Average (Min.-Max) }\end{array}$ \\
\hline Temperature $\left({ }^{\circ} \mathrm{C}\right)$ & Average (Min.-Max) & $2.82(-14.44-29.72)$ & $21.72(4.83-36.94)$ \\
Temperature $\left({ }^{\circ} \mathrm{C}\right)$ TB Area & $-2.75(-27.50-12.80)$ & $-0.35(-22.20-28.80)$ & $19.55(0.70-35.80)$ \\
Precipitation $(\mathrm{mm})$ & $1.71(0.00-20.83)$ & $0.91(0.00-18.54)$ & $3.13(0.00-30.48)$ \\
Precipitation $(\mathrm{mm})$ TB Area & $1.49(0.00-22.35)$ & $0.78(0.00-12.70)$ & $2.02(0.00-24.64)$ \\
Humidity $(\%)$ & $85.92(27.00-100.00)$ & $66.07(4.00-100.00)$ & $62.44(10.00-100.00)$ \\
Solar Radiation $\left(\mathrm{W} / \mathrm{m}^{2}\right)$ & $24.29(0.00-347.00)$ & $79.99(0.00-1040.00)$ & $149.95(0.00-1170.00)$ \\
Evapotranspiration $(\mathrm{mm})$ & $0.03(0.00-1.02)$ & $0.18(0.00-2.29)$ & $0.38(0.00-3.30)$ \\
\hline
\end{tabular}

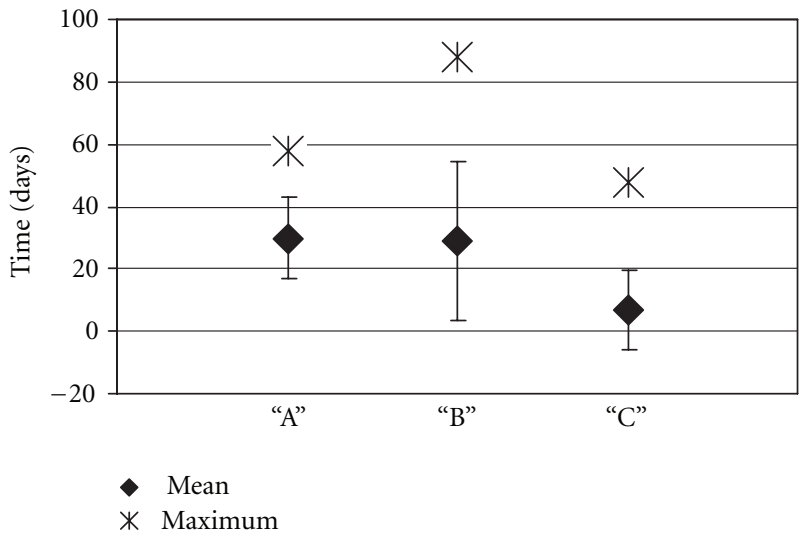

FIGURE 4: The overall mean and maximum survival time (persistence) of $M$. bovis in all substrates exposed to natural environmental conditions in Fall/Winter "A", Winter/Spring "B", and Spring/Summer "C". Error bars represent standard deviation.

contamination across all sampling periods ("A": $\chi^{2}=20.28$, $P<.01$; “B": $\chi^{2}=10.29, P<.01$; “C": $\left.\chi^{2}=4.7, P<.05\right)$.

3.2. Environmental Conditions. The coldest sampling period was the Fall/Winter "A", Winter/Spring "B" was intermediate, and Spring/Summer "C" was the warmest with the greatest amount of precipitation, highest solar radiation, and greatest degree of evapotranspiration (Table 1). Daily average temperatures and daily average rainfall recorded at the Michigan Automated Weather Network weather station in Hawk City, Presque Isle Country, Mich, USA in the endemic bovine TB area over the same time periods revealed similar trends in temperature patterns (highs and lows) and similar patterns of precipitation. As expected, the average daily temperature at the northern weather station in the bovine TB endemic region was approximately 3 degrees lower across all sampling periods (Table 1).

3.3. Persistence of $M$. bovis in the Environment. M. bovis persisted in substrates exposed to environmental conditions for an average of one month in cool Fall/Winter and Winter/Spring conditions and for an average of 7 days in warmer Spring/Summer conditions (Table 2). Both the time from inoculation to the last positive M. bovis sample and

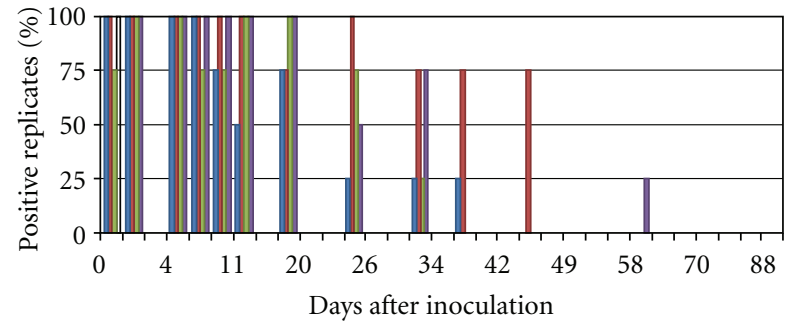

(a)

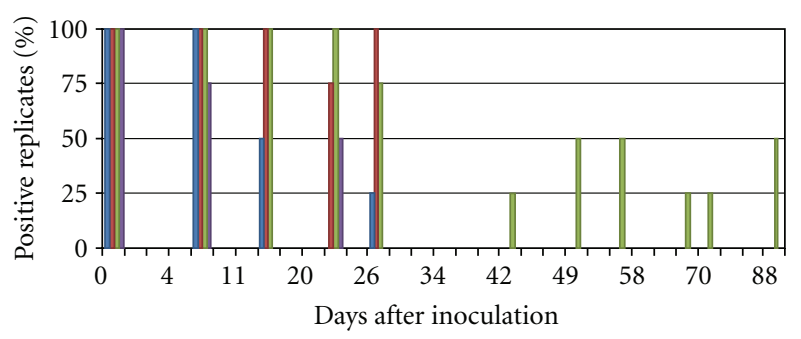

(b)

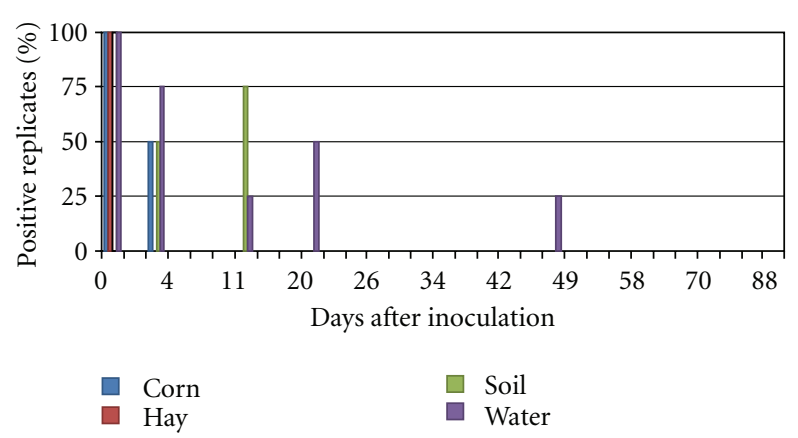

(c)

FIGURE 5: The percent ( $n=4$ of each substrate) of $M$. bovis positive replicates of corn, hay, soil, and water at each sampling point in Fall/Winter (a), Winter/Spring (b), and Spring/Summer (c).

the time from inoculation to first negative after last positive $M$. bovis sample are presented. The average and maximum survival times were lowest in the Spring/Summer sampling period "C". The maximum survival time across all substrate types and sampling seasons was recorded in a soil sample 
TABLE 2: Duration of M. bovis persistence in the environment on corn, hay, water and soil samples in season "A", "B" and "C".

\begin{tabular}{|c|c|c|c|c|}
\hline & \multicolumn{2}{|c|}{ Days to last positive } & \multicolumn{2}{|c|}{ Days to 1st negative after last positive } \\
\hline & Mean (standard deviation) & Maximum (days) & Mean (standard deviation) & Maximum (days) \\
\hline \multicolumn{5}{|c|}{ Fall/Winter "A" } \\
\hline Corn & $24.00(11.75)$ & 37.00 & $29.75(13.94)$ & 43.00 \\
\hline Hay & $41.50(3.00)^{*}$ & 43.00 & $54.25(7.5)^{*}$ & 58.00 \\
\hline Soil & $21.75(5.32)$ & 28.00 & $28.75(6.18)^{*}$ & 37.00 \\
\hline Water & $32.25(18.23)$ & 58.00 & $38.50(14.80)$ & 58.00 \\
\hline All samples & $29.88(12.88)$ & 58.00 & $37.81(14.59)$ & 58.00 \\
\hline \multicolumn{5}{|c|}{ Winter/Spring "B" } \\
\hline Corn & $15.25(7.89)^{*}$ & 26.00 & $24.50(12.12)^{*}$ & 42.00 \\
\hline Hay & $26.00(0.00)$ & 26.00 & $42.00(0.00)$ & 42.00 \\
\hline Soil & $62.75(30.63)^{*}$ & 88.00 & $68.25(23.41)^{*}$ & 88.00 \\
\hline Water & $12.25(10.50)^{*}$ & 21.00 & $18.25(9.39)^{*}$ & 26.00 \\
\hline All samples & $29.06(25.56)$ & 88.00 & $38.25(23.61)$ & 88.00 \\
\hline \multicolumn{5}{|c|}{ Spring/Summer "C" } \\
\hline Corn & $1.50(1.73)$ & 3.00 & $7.00(4.62)$ & 11.00 \\
\hline Hay & $0.00(0.00)$ & 0.00 & $3.00(0.00)$ & 3.00 \\
\hline Soil & $8.25(5.50)$ & 11.00 & $15.75(8.50)$ & 20.00 \\
\hline Water & $17.75(22.01)^{*}$ & 48.00 & $23.50(22.05)^{*}$ & 53.00 \\
\hline All samples & $6.88(12.48)$ & 48.00 & $12.31(13.53)$ & 53.00 \\
\hline
\end{tabular}

${ }^{*}$ Significant difference in means (ANOVA) among substrate type Bonfferoni $P \leq .05$.

in the Winter/Spring "B" period. The soil sample was $M$. bovis positive at the final sampling point of 88 days. The shortest survival period was recorded in a hay sample in the Spring/Summer "C" period. The sample was positive at time 0 but negative at the 1 st sampling point at 3 days. The mean and maximum survival time in each substrate type in each season is presented in Figure 3. The overall mean and maximum survival time across all substrate types in each season is presented in Figure 4.

The number of M. bovis positive replicate samples, and the number of $M$. bovis colonies recovered per sample, drop off quickly over the first 7 to 14 days of exposure to environmental conditions. The isolation of $M$. bovis from substrates exposed to environmental conditions was more intermittent after 14 days and positive samples were often identified based on the isolation of less than $5 \mathrm{M}$. bovis colonies per $100 \mu \mathrm{L}$ of sample. The percent of M. bovis positive replicates recorded at each sampling point and the number of colony forming units isolated are displayed in Figures 5 and 6.

3.4. Effects of Nonseasonal Factors on the Persistence of $M$. bovis in the Environment. The effect of substrate type on the persistence of $M$. bovis in the environment was variable. In the Spring/Summer period "C" survival was significantly longer in water. In the Fall/Winter "A" period survival appeared to be significantly longer in hay, and in the Winter/Spring "B" period survival appeared to be significantly longer in soil. Survival probability curves for M. bovis in soil, corn, hay and water across all seasons are illustrated in Figure 7. The curves appear similar and log rank statistics confirm that the survival curves for the different substrates types are not significantly different from one another $\left(\chi^{2}=5.03, P=.17\right)$. Among all seasons there is no significant difference in the survival of $M$. bovis in one sample type versus another.

No significant associations were found between the bovine TB status of a sample and whether or not it was sterilized before M. bovis inoculation. The placement of the inoculated samples in shade or direct sunlight did not have a statistically significant effect on M. bovis survival, but the mean survival time was longer across all samples and seasons in those placed under shade. The difference in mean survival time in the shaded and nonshaded samples approached significance in the Fall/Winter "A" and Spring/Summer "C" seasons (Table 3 ).

3.5. Effects of Season on M. bovis Persistence in the Environment. Figure 8 illustrates the survival probability curves of $M$. bovis organisms exposed to environmental conditions in Fall/Winter "A", Winter/Spring "B", and Spring/Summer "C". The log rank statistics were associated with highly significant differences (chi-square $=19.88, P<.0001$ ) for between season probabilities. An analysis of maximum likelihood estimates when other covariates (shade/nonshade, substrate type, sterilized/nonsterilized substrates, and interaction between shade/nonshade and season) were added to the model makes it clear that it is the season that drives the difference in the survival probability (Table 4).

3.6. Effects of Weather on M. bovis Persistence in the Environment Across Seasons. The Cox's proportional hazard regression model, used to determine the relative influence of various weather related factors that together contribute to 
TABLE 3: Mean duration in days of M. bovis persistence in the environment on corn, hay, water and soil samples in season "A", "B", and "C" in shaded and nonshaded conditions.

\begin{tabular}{|c|c|c|c|c|}
\hline & Shade mean (Std. Dev.) & No shade mean (Std. Dev.) & $t$ statistic & $P$-value \\
\hline Fall/Winter "A" & $12.79(12.27)$ & $9.58(9.38)$ & -1.68 & .10 \\
\hline Winter/Spring "B" & $18.40(20.65)$ & $17.41(20.48)$ & -0.20 & .84 \\
\hline Spring/Summer "C" & $7.18(12.52)$ & $2.38(4.03)$ & -1.48 & .15 \\
\hline
\end{tabular}

TABLE 4: Cox's proportional hazard regression model for persistence of M. bovis over three different sampling periods (Fall/Winter "A", Winter/Spring "B" and Spring/Summer "C") or seasons.

\begin{tabular}{lccc}
\hline Risk factor & Parameter estimate & Standard error & Hazard ratio (95\% confidence interval) \\
\hline Season A & -2.13 & 0.49 & $0.12(0.05-0.32)$ \\
Season B & -1.94 & 0.49 & $0.14(0.5-0.38)$ \\
Season C & 0 & - & - \\
Sample Type & -0.28 & 0.16 & $0.76(0.55-1.05)$ \\
Sterilized & -0.50 & 0.35 & $0.61(0.31-1.20)$ \\
Shade & -0.68 & 0.33 & $0.51(0.26-1.05)$ \\
\hline
\end{tabular}

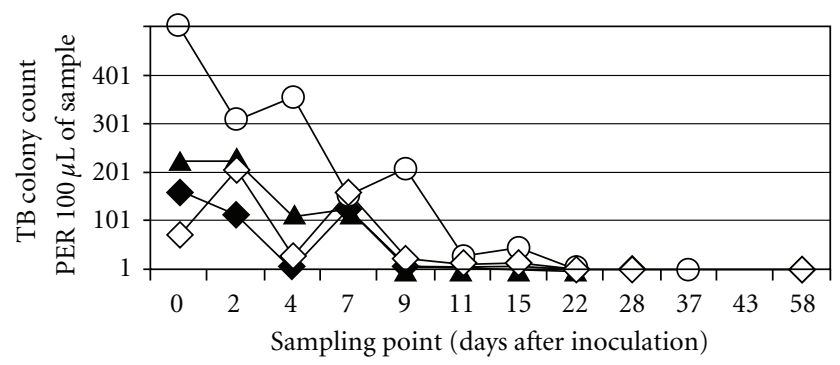

(a)

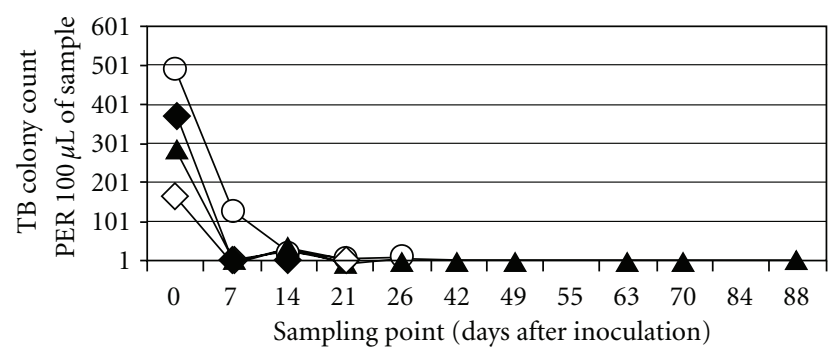

(b)

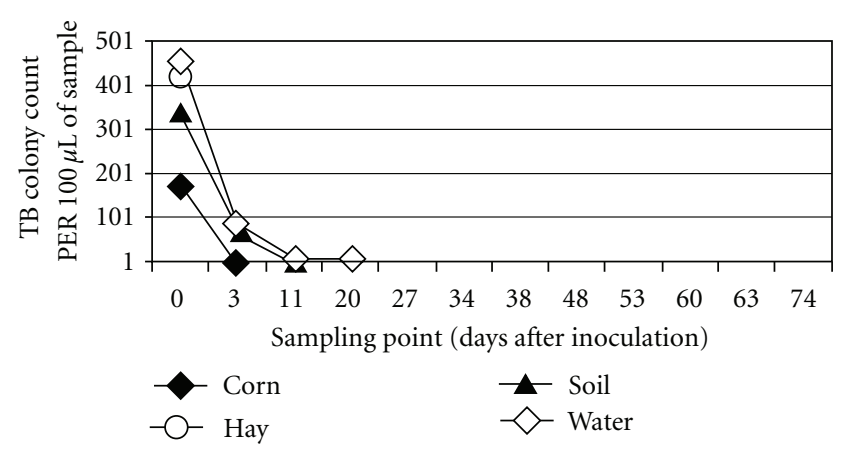

(c)

FIGURE 6: The average number of $M$. bovis colony forming units isolated on solid media from $100 \mu \mathrm{L}$ of processed sample (corn, hay, soil and water) over time in Fall/Winter (a), Winter/Spring (b), and Spring/Summer (c). seasonal differences in the environmental persistence of $M$. bovis, revealed that temperature is the most influential factor in $M$. bovis survival. A number of the weather-related factors recorded throughout the sampling periods were significantly associated with the survival of $M$. bovis in the environment, however, many of these factors correlated with one another. The univariable hazard ratios and 95\% confidence intervals for the weather-related factors tested are presented in Table 5. Although all of these variables were significant at the $P<.15$ level, evapotranspiration and solar radiation were removed due to redundancy. The final multivariable Cox proportional hazard regression model is presented in Table 6.

\section{Discussion}

Mycobacterium bovis is an obligate intracellular pathogen but it has been shown to survive in the environment, outside a host, for substantial periods of time under favorable conditions [11-15, 18-24]. Although all of these studies demonstrated the persistence of $M$. bovis in the environment, their experimental nature, the use of high bacterial loads, and the variability of results have generally led to the conclusion that the environmental persistence of $M$. bovis does not play a significant role in the epidemiology of bovine TB through the indirect transmission of the pathogen among or between susceptible species.

The outbreak of bovine TB in northeast Michigan, and the establishment of a wildlife reservoir (white-tailed deer) for $M$. bovis in the region, has renewed interest in the characterization of $M$. bovis persistence in the environment and its role in the epidemiology of bovine TB in North America. This study clearly demonstrates that the Michigan strain of $M$. bovis persists in the environment under typical Michigan weather conditions. The study mimicked, to the greatest degree possible, the natural conditions under which M. bovis would be deposited on substrates in the environment and the weather to which the organisms would be exposed. Since 
TABLE 5: Univariable analysis of the influence of each weather related variable on the hazard (survival) of M. bovis in the environmental using the score test in Cox regression.

\begin{tabular}{lcc}
\hline Weather factor (average) & Score Chi-square & $P$-value \\
\hline Solar Radiation & 7.50 & .0062 \\
Humidity & 2.46 & .1165 \\
Temperature & 9.85 & .0017 \\
Precipitation & 5.44 & .0197 \\
Evapotranspiration & 17.19 & $<.0001$ \\
\hline
\end{tabular}

TABLE 6: Multivariable Cox proportional hazards model of weather related factors associated with the survival of $M$. bovis in the environment.

\begin{tabular}{lccc}
\hline Risk factor & Parameter estimate & Standard error & Hazard ratio (95\% confidence interval) \\
\hline Temperature* & 0.06 & 0.02 & $1.06(1.02-1.10)$ \\
\hline
\end{tabular}

${ }^{*}$ Results of purposeful selection of covariate and modified stepwise modeling in Cox regression. Only temperature remains in the model.

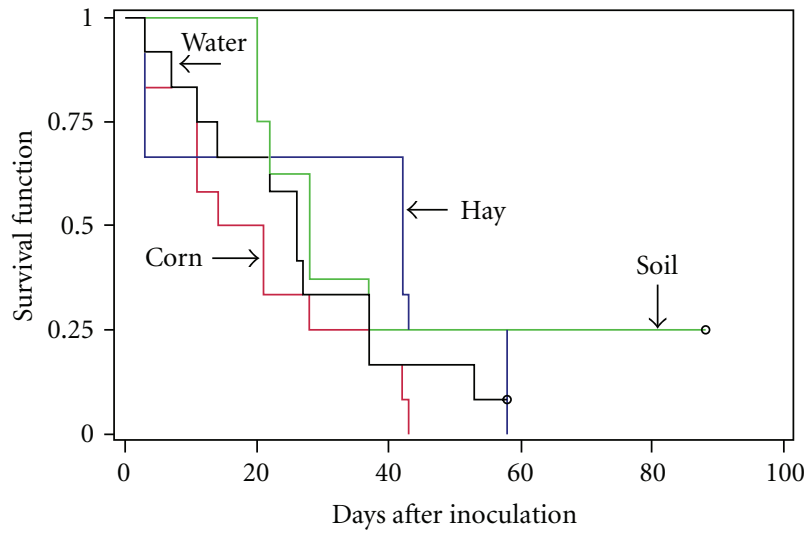

FIGURE 7: Survival probabilities of M. bovis in water, corn, hay, and soil across all seasons tested.

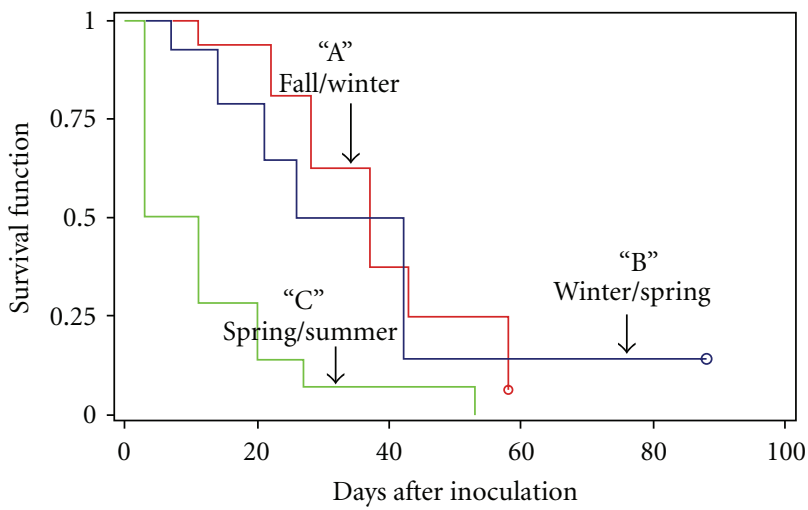

Figure 8: Survival probabilities of $M$. bovis exposed to natural environmental conditions in "A", "B", and "C" sampling periods.

the study site was approximately 200 miles to the south of the endemic bovine TB area in Michigan, basic indicators of environmental conditions (average daily temperature and precipitation) were compared between the two areas by using data from a weather station in the Michigan Automated Weather Network located in Hawk City, Presque Isle County. Daily temperature changes and precipitation at the study site and the northern Michigan weather station were graphed and compared visually. The temperature cycles (periods of high and low temperatures) and patterns of precipitation recorded at the northern Michigan weather station mimicked those observed at the study site, however, the temperature in the northern $\mathrm{TB}$ endemic area was on average of $3^{\circ} \mathrm{C}$ cooler throughout the sampling periods. These slightly cooler temperatures in the north could be expected to support slightly longer persistence of $M$. bovis in the environment.

4.1. The Persistence of $M$. bovis in the Environment. The general pattern of $M$. bovis persistence in the environment, across all seasons and in all substrate types, was an initial decline in the numbers of recoverable bacilli over a 7 -to14-day period and then the persistence of small numbers of $M$. bovis bacilli for up to 4 to 12 weeks. The persistence of the Michigan strain of M. bovis on corn, hay, soil, and water recorded in this natural environmental exposure study confirms the findings of laboratory-based studies conducted earlier in which the Michigan strain of M. bovis was found to persist for up to 12 weeks on feed (hay, corn, sugar beets, apples, carrots, and potatoes) stored at $46^{\circ} \mathrm{F}$ and $0^{\circ} \mathrm{F}$ and for shorter periods when stored at $75^{\circ} \mathrm{F}$ [25]. The similarity between the findings of the laboratory-based studies on $M$. bovis persistence and this one, in which the experimentally infected substrates were exposed to natural environmental conditions, indicates that failure to detect $M$. bovis in the environment in known areas of $M$. bovis transmission is likely associated with the highly clustered spatial distribution of contaminated substrates under natural conditions and the inability to pinpoint the exact location of a $M$. bovis contaminated substrate for sampling, and not the inability of $M$. bovis to survive in the environment in a viable state.

The results of early studies performed in Europe and designed to characterize the persistence of $M$. bovis in the environment, have been scrutinized due to the large numbers of $M$. bovis bacilli used to experimentally inoculate of substrates [18-21]. In this study the initial bacterial load used to inoculate the substrates was $50,000 \mathrm{CFU}$ of $M$. bovis. This amount of $M$. bovis is more than the minimum infective oral dose of $M$. bovis established through experimental studies 
with the Michigan strain of $M$. bovis in both cattle $(5,000$ CFU) and white-tailed deer (300 CFU) [26, 27]. The 50,000 CFU inoculums used is thought to be indicative of the amount of M. bovis that could be deposited by a bovine TBinfected and shedding animal. The 50,000 CFU inoculum is smaller than the number of $M$. bovis bacilli recorded in the exudates from a lesion in an infected brush-tailed possum in New Zealand $\left(5 \times 10^{8} \mathrm{CFU} / \mathrm{mL}\right)$ [28] but larger than number of bacilli (approximately $70 \mathrm{CFU}$ ) detected in the nasal mucous of an experimentally infected cow [29]. The amount of $M$. bovis used to inoculate substrates is believed to be relevant both in terms of the probability of contamination of environmental substrates through shedding of $M$. bovis from an infected animal and the likelihood of ingestion of M. bovis bacilli present in the environment by a susceptible host. This is particularly true in the 1st 7 days of exposure of $M$. bovis to environmental conditions when the number of CFUs recovered from the experimentally inoculated samples remained high.

4.2. Factors Influencing the Persistence of $M$. bovis in the Environment. This study was designed to characterize the persistence of $M$. bovis in a number of substrates exposed to natural environmental conditions over a 12 -month period. A review of the literature indicated the necessity to supplement the 12-month study with a number of seasonal experiments designed to capture the effects on the persistence of $M$. bovis under different weather conditions. The persistence of $M$. bovis during the Michigan Spring/Summer season (May 20th to August 2nd) was significantly shorter than the persistence recorded in the Fall/Winter season (November 8th to January 6th) and Winter/Spring season (February 4 to May 3). The Spring/Summer season was associated with higher average daily temperatures, greater intensity of solar radiation, and higher loss of moisture through evapotranspiration. These findings are in agreement with those reported elsewhere in which an increase in temperature and a loss of moisture were found to be associated with a decrease in the persistence of M. bovis in the environment [12-14, 22].

Other factors, including substrate type, did not significantly affect the pattern of $M$. bovis persistence. The detection of $M$. bovis in a soil sample at 88 days (the final sampling point) in the Winter/Spring sampling period is in agreement with other studies that recorded the longest survival of $M$. bovis in cool, moist soil that presumably protects the bacilli from desiccation and provides an organic environment that supports its persistence $[12,19,20]$. Detection of $M$. bovis in a water sample at 48 days in the Spring/Summer sampling period, in which all other substrate types were negative at 20 days, indicates that even in the presence of high temperatures and intense solar radiation, viable $M$. bovis can persist under moist conditions.

The sterilization of substrates before M. bovis inoculation did not affect the persistence of $M$. bovis, but it did significantly decrease the occurrence of contaminated bacterial cultures. Presterilized substrates were not used exclusively in this study because decreased survival of $M$. bovis in sterile substrates has been reported $[12,15]$.
The location of the M. bovis inoculated substrates under shade had an effect on persistence. The mean survival time was longer for samples under shade than those exposed to direct sunlight. This was true in the Fall/Winter season and the Spring/Summer season but not in the Winter/Spring season. This apparent lack of a protective effect of shade during the Winter/Spring season is likely due to the fact that the cloth used to cover the "shaded" samples during this sampling period was severely damaged by wind and removed. It was replaced before the final Spring/Summer sampling period.

Survival analysis was used to evaluate the impact of weather over the three seasons on the persistence of $M$. bovis in the environment. Univariate analysis using Cox's proportional hazard regression indicated that the survival probability or persistence of $M$. bovis was decreased as temperatures increased, solar radiation intensified, and evapotranspiration (a measure of moisture loss from a system) increased. The effects of average precipitation and percent humidity lost their significance in the presence of temperature. Since solar radiation and evapotranspiration are both directly related to temperature, the final conclusion was that temperature drives the seasonal effect seen in $M$. bovis persistence in the environment.

4.3. Implications of the Persistence of $M$. bovis in the Environment. The evidence that viable $M$. bovis persists in the Michigan environment under near natural conditions has significant implications on the efforts to control and eventually eliminate the occurrence of bovine $\mathrm{TB}$ in the region. Authors have argued when examining other systems of bovine TB in other parts of the world that the conditions that contribute to $M$. bovis persistence in the environment also make the organisms inaccessible to susceptible hosts $[13,30]$. This is not the case in Michigan. The types of substrates tested (soil, water, hay, and corn) are present in and around cattle farms in northeast Michigan. Additionally, white-tailed deer have access to these substrates on many cattle operations. Although many feed piles are exposed to sunlight and Summer temperatures, there are periods of the year throughout the region in which low temperatures, cloud cover, and the location of feed and water sources under the cover of a forest canopy or otherwise protected from the elements, would facilitate the long-term (4-12 weeks) survival of $M$. bovis bacilli deposited by a bovine TB-infected animal.

The elimination of feeding and baiting sites for whitetailed deer and other wildlife should remain a component of the efforts to reduce bovine TB prevalence in this population and part of the management recommendations designed to reduce deer-to-deer bovine $\mathrm{TB}$ transmission events. Bovine TB eradication programs designed to eliminate the occurrence of bovine TB in cattle must consider $M$. bovis contaminated feed or water as a possible route for the indirect transmission of bovine TB between infected whitetailed deer and cattle. Farm biosecurity measures focused on the elimination of the possibilities of cross contamination of feed and water sources should be added to the current protocols designed to eliminate cattle-to-cattle and the direct 
transmission of bovine TB. Specifically, cattle should be fenced out of open water sources, and they should be provided an alternative source of water. Cattle should not be fed hay on the ground in the woods or in pasture adjacent to wooded areas and crops fields known to attract white-tailed deer. The programs designed to encourage the fencing of feed storage areas should continue, but emphasis should also be placed on the fencing and protection of cattle feeding areas.

\section{Conclusions}

The data clearly indicate that there is a real potential for the indirect transmission of $M$. bovis among and between cattle and white-tailed deer populations in Michigan. Persistence of M. bovis can be expected to be longer in cooler seasons. Practices that facilitate the cross contamination of substrates by infected and susceptible animals should be restricted at all times but especially during the cooler seasons of the year.

Failure to isolate $M$. bovis from environmental substrates collected from areas with known bovine TB transmission, is likely due to the inability to pinpoint the exact location of environmental contamination for sampling and less to do with the persistence of the $M$. bovis bacilli in the environment. Difficulties in isolating viable M. bovis from environmental substrates due to the limitations of sample processing and mycobacterial culture will also continue to hinder the accurate assessment of the quantity of viable $M$. bovis organisms in the environment. However, this study indicates that the organisms do persist over a time period that would allow a susceptible animal to become exposed and infected with $M$. bovis from an environmental source. Indirect transmission of $M$. bovis plays a role in the interspecies transmission of bovine $\mathrm{TB}$ and will continue to hinder programs designed to eliminate the disease if not addressed.

The recorded persistence of M. bovis in the Michigan environment under natural weather conditions strongly suggests the potential contribution of indirect means to the transmission of bovine TB in the region. These data supplement those produced through experimental M. bovis disease transmission studies that have proven the feasibility of indirect transmission of $M$. bovis among and between cattle and white-tailed deer. They also support the analyses of observational data on M. bovis infection in cattle and white-tailed deer in Michigan that indicate the importance of indirect transmission in the interspecies transmission of M. bovis in the region.

\section{Acknowledgments}

This research was supported by the United States Department of Agriculture, National Research Institute (Bovine Tuberculosis-Special grant), the Michigan Department of Agriculture, Animal Industry Division, the United States Department of Agriculture, Animal and Plant Health Inspection Service, Wildlife Services, the Center for Comparative Epidemiology, and the Department of Large Animal Clinical Sciences, College of Veterinary Medicine, Michigan
State University. Joseph Hattey, Christie Sampson, and Kyle Kucher provided laboratory support and assistance with data management. John Baker provided mentorship and overall guidance.

\section{References}

[1] D. J. O’Brien, S. M. Schmitt, S. D. Fitzgerald, D. E. Berry, and G. J. Hickling, "Managing the wildlife reservoir of Mycobacterium bovis: the Michigan, USA, experience," Veterinary Microbiology, vol. 112, no. 2-4, pp. 313-323, 2006.

[2] S. M. Schmitt, S. D. Fitzgerald, T. M. Cooley et al., "Bovine tuberculosis in free-ranging white-tailed deer from Michigan," Journal of Wildlife Diseases, vol. 33, no. 4, pp. 749-758, 1997.

[3] D. L. Whipple, J. L. Jarnagin, and J. B. Payeur, "DNA fingerprinting of Mycobacterium bovis isolates from animals in northeast Michigan," in Proceedings of the IX International Symposium of the World Association of Veterinary Laboratory Diagnosticians, World Association of Veterinary Laboratory Diagnosticians, College Station, Tex, USA, 1999.

[4] T. J. DeLiberto, K. C. Vercauteren, and G. W. Witmer, "The ecology of Mycobacterium bovis in Michigan," in Activities Report and Conference Proceedings, Michigan Bovine Tuberculosis Eradication Project, Lansing, Mich, USA, 2004.

[5] G. Frye, "Bovine tuberculosis eradication: the program in the United States," in Mycobacterium bovis Infection in Animals and Humans, C. O. Thoen and J. H. Steele, Eds., pp. 119-129, Iowa State University Press, Ames, Iowa, USA, 1995.

[6] G. J. Hickling, "Dynamics of bovine tuberculosis in wild white-tailed deer in Michigan," Wildlife Division Report No. 3363, Michigan Department of Natural Resources, 2002.

[7] R. Miller, J. B. Kaneene, S. D. Fitzgerald, and S. M. Schmitt, "Evaluation of the influence of supplemental feeding of whitetailed deer (Odocoileus virginianus) on the prevalence of bovine tuberculosis in the Michigan wild deer population," Journal of Wildlife Diseases, vol. 39, no. 1, pp. 84-95, 2003.

[8] A. E. Fine, D. J. O'Brien, S. R. Winterstein, and J. B. Kaneene, "An effort to isolate Mycobacterium bovis from environmental substrates during investigations of bovine tuberculosis transmission sites (cattle farms and wildlife areas) in Michigan, USA," submitted.

[9] G. Witmer, A. E. Fine, J. Glonfriddo et al., "Epizootiologic survey of Mycobacterium bovis in wildlife and farm environments in northern Michigan," Journal of Wildlife Diseases, vol. 46, no. 2, pp. 368-378, 2010.

[10] J. B. Kaneene, C. S. Bruning-Fann, L. M. Granger, R. Miller, and B. A. Porter-Spalding, "Environmental and farm management factors associated with tuberculosis on cattle farms in northeastern Michigan," Journal of the American Veterinary Medical Association, vol. 221, no. 6, pp. 837-842, 2002.

[11] T. W. A. Little, P. F. Naylor, and J. W. Wilesmith, "Laboratory study of Mycobacterium bovis infection in badgers and calves," Veterinary Record, vol. 111, no. 24, pp. 550-557, 1982.

[12] B. J. Duffield and D. A. Young, "Survival of Mycobacterium bovis in defined environmental conditions," Veterinary Microbiology, vol. 10, no. 2, pp. 193-197, 1984.

[13] R. Jackson, G. W. de Lisle, and R. S. Morris, "A study of the environmental survival of Mycobacterium bovis on a farm in New Zealand," New Zealand Veterinary Journal, vol. 43, pp. 346-352, 1995. 
[14] M. Tanner and A. L. Michel, "Investigation of the viability of $M$. bovis under different environmental conditions in the Kruger National Park," Onderstepoort Journal of Veterinary Research, vol. 66, no. 3, pp. 185-190, 1999.

[15] J. S. Young, E. Gormley, and E. M. H. Wellington, "Molecular detection of Mycobacterium bovis and Mycobacterium bovis BCG (Pasteur) in soil," Applied and Environmental Microbiology, vol. 71, no. 4, pp. 1946-1952, 2005.

[16] A. E. Fine, The role of indirect transmission in the epidemiology of bovine tuberculosis in cattle and white-tailed deer in Michigan, Ph.D. dissertation, Michigan State University, East Lansing, Mich, USA, 2006, p. 157.

[17] P. T. Kent and G. P. Kubica, Public Health Mycobacteriology: A Guide for Level III Laboratory, U.S. Department of Health and Human Services, Centers for Disease Control, Public Health Service, Atlanta, Ga, USA, 1985.

[18] S. R. Williams and W. A. Hoy, "The viability of B. tuberculosis (bovinus) on pasture land, in stored faeces and in liquid manure," Journal of Hygiene, vol. 30, pp. 413-419, 1930.

[19] E. C. G. Maddock, "Studies on the survival time of the bovine tubercle bacillus in soil, soil and dung, in dung and on grass, with experiments on the preliminary treatment of infected organic matter and the cultivation of the organism," Journal of Hygiene, vol. 33, pp. 103-117, 1933.

[20] E. C. G. Maddock, "Further studies on the survival time of the bovine tubercle bacillus in soil, soil and dung, in dung and on grass, with experiments on feeding guinea-pigs and calves on grass artificially infected with bovine tubercle bacilli," Journal of Hygiene, vol. 34, pp. 372-379, 1934.

[21] E. C. G. Maddock, "Experiments on the infectivity for healthy calves on bovine tubercle bacilli discharged in dung upon pasture. Part 1. From tubercular calves fed with emulsion of tubercle bacilli 1934-1935. Part 2. From tubercular cows passing tubercle bacilli in their dung 1935-1936," Journal of Hygiene, vol. 36, pp. 594-601, 1936.

[22] C. Wray, "Survival and spread of pathogenic bacteria of veterinary importance within the environment," Veterinary Bulletin, vol. 45, pp. 543-550, 1975.

[23] D. J. van Donsel and E. P. Larkin, "Persistence of Mycobacterium bovis BCG in soil and on vegetables spray-irrigated with Sewage effluent and sludge," Journal of Food Protection, vol. 40, no. 3, pp. 160-163, 1977.

[24] E. Mitscherlich and E. H. Marth, "Mycobacterium bovis," in Microbial Survival in the Environment: Bacteria and Rickettsiae Important in Human and Animal Health, pp. 235-242, Springer, Berlin, Germany, 1984.

[25] M. V. Palmer and D. L. Whipple, "Survival of Mycobacterium bovis on feedstuffs commonly used as supplemental feed for white-tailed deer (Odocoileus virginianus)," Journal of Wildlife Diseases, vol. 42, no. 4, pp. 853-858, 2006.

[26] M. V. Palmer, W. R. Waters, and D. L. Whipple, "Lesion development in white-tailed deer (Odocoileus virginianus) experimentally infected with Mycobacterium bovis," Veterinary Pathology, vol. 39, no. 3, pp. 334-340, 2002.

[27] M. V. Palmer, W. R. Waters, and D. L. Whipple, "Investigation of the transmission of Mycobacterium bovis from deer to cattle through indirect contact," American Journal of Veterinary Research, vol. 65, no. 11, pp. 1483-1489, 2004.

[28] B. L. Smith, "Tuberculosis in the opossum," New Zealand Veterinary Journal, vol. 20, p. 199, 1972.

[29] T. McCorry, A. O. Whelan, M. D. Welsh et al., "Shedding of Mycobacterium bovis in the nasal mucus of cattle infected experimentally with tuberculosis by the intranasal and intratracheal routes," Veterinary Record, vol. 157, no. 20, pp. 613618, 2005.

[30] R. S. Morris, D. U. Pfeiffer, and R. Jackson, “The epidemiology of Mycobacterium bovis infections," Veterinary Microbiology, vol. 40, no. 1-2, pp. 153-177, 1994. 

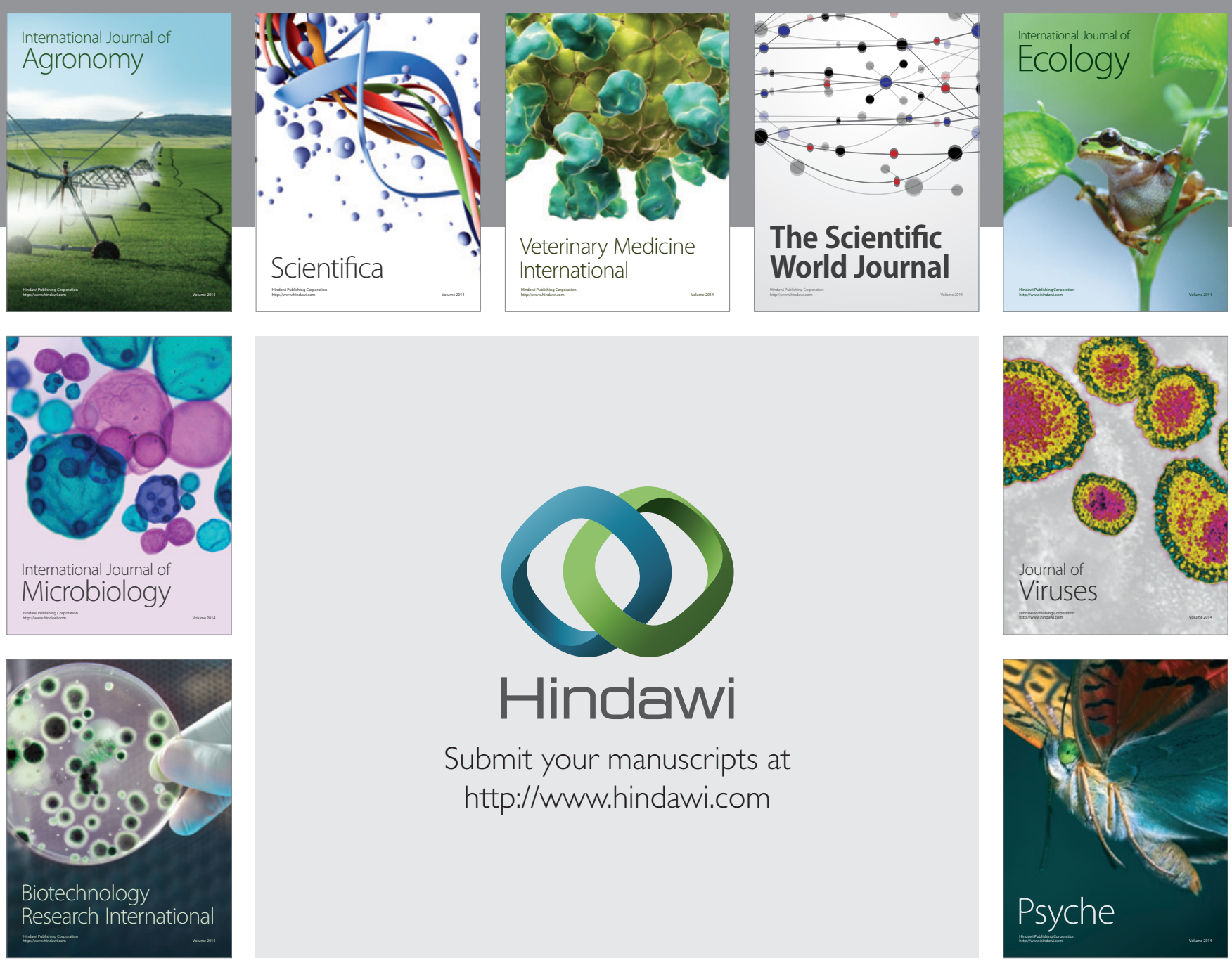

Submit your manuscripts at

http://www.hindawi.com
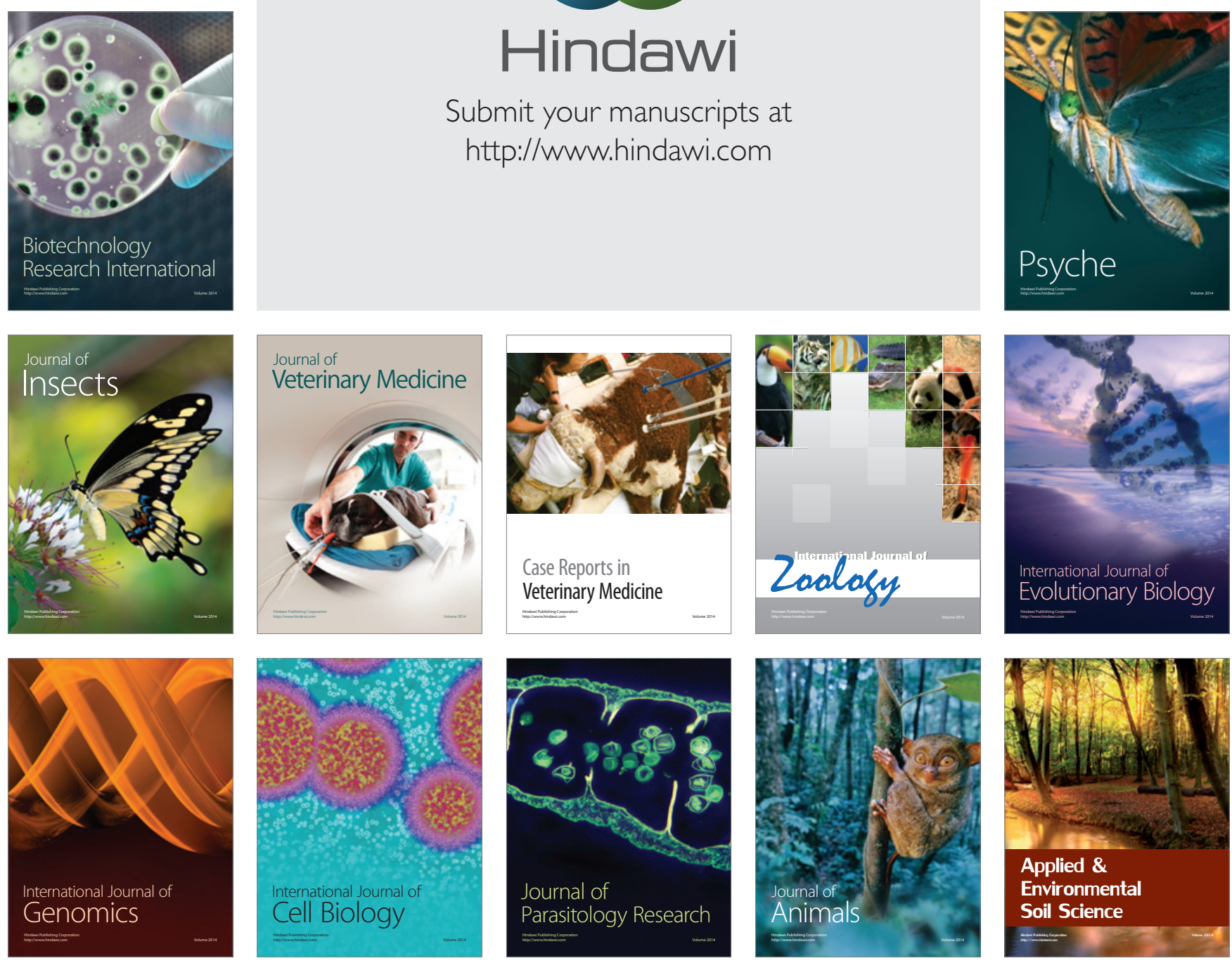